\title{
Adverse drug reactions of non-opioid and opioid analgesics reported to Croatian national authority from 2007 to 2014
}

\author{
${ }^{1}$ Laboratory for Pain Research, University \\ of Split School of Medicine, Split \\ Croatia, ${ }^{2}$ Agency for Medicinal Products \\ and Medical Devices, Zagreb, Croatia \\ ${ }^{3}$ Department for Development, Research \\ and Health Technology Assessment, \\ Agency for Quality and Accreditation \\ in Health Care and Social Welfare, \\ Zagreb, Croatia
}

Petra Sunara ${ }^{1}$, Darko Krnic ${ }^{2}$, Livia Puljak ${ }^{1,3}$

$$
\begin{aligned}
& \text { Correspondence: } \\
& \text { livia.puljak@mefst.hr } \\
& \text { Tel.: + } 38521557807 \\
& \text { Fax.: + 38521557811 }
\end{aligned}
$$

Received: 7 June 2017

Accepted: 30 October 2017

Key words: Adverse drug reactions • Opioids • Non-opioids • Analgesics • Croatia.

\begin{abstract}
Objective. Adverse drug reactions (ADRs) are commonly observed in the health services because of system weaknesses and individual errors. Analgesics are widely used and it can be expected that with the increased use one can expect increased number of ADRs of analgesics. The aim of this study was to analyze ADRs of non-opioid and opioid analgesics reported to the Croatian Agency for Medicinal Products and Medical Devices (HALMED) from 2007 to 2014. Methods. HALMED provided data on generic drug name, year of the ADR report, type of report, institution, reporting person, patient's age, sex and ADR type. Results. In the analyzed period 796 ADRs of analgesics were reported, of which 367 (46\%) were serious ADRs. Number of ADR reports was continuously increasing during the analyzed period. There were 20 analgesics that had $\geq 5$ reports, making 597 (75\%) of all ADR reports for analgesics. The most common adverse reaction reports of those 20 analgesics referred to individual drugs ( $n=16 ; 80 \%)$. Most of the ADR reports were filed by physicians ( $n=257 ; 43 \%)$, followed by pharmacists ( $n=252 ; 42 \%)$. Most side effects $(n=572 ; 96 \%)$ were reported spontaneously through appropriate forms by patients or health professionals. ADRs were most commonly reported in women $(n=352 ; 59 \%)$ and most of them have occurred in adults $(n=354$; $59 \%$ ). The most common ADRs of opioid and non-opioid analgesics have been reported on the skin and mucous membranes. Most serious ADRs were result of action of opioid analgesics. Conclusion. Number of ADR reports in Croatia is continuously increasing and a considerable number of them refers to serious ADRs. To keep better track of medications and ADRs it is necessary to educate and encourage health professionals and patients in reporting side effects.
\end{abstract}

\section{Introduction}

Adverse drug reactions (ADRs) are commonly observed in the health services because of system weaknesses and individual errors (1). Public health burden of ADRs associated with drug use among non-hospitalized patients in community has been difficult to estimate, but the problem is being documented, it is considerable and it is expected that it will increase (2). ADRs contribute to a loss of public confidence, increase patient morbidity and mortality, as well as raise health care costs (2). Analgesics are widely used and it can be expected that with the increased use one can expect increased number of ADRs of analgesics. Large increases in the use of both opioid and non-opioid analgesics were observed worldwide (3-8). 
Some medications such as aspirin, paracetamol and ibuprofen belong to the first step of the World Health Organization (WHO) pain treatment ladder, and they are used on a daily basis as an over-the-counter (OTC) medications by millions of individuals. Even though they are generally well tolerated, even apparently minor ADRs can appear significant from a public health point of view (9). These trends underscore the need for ongoing surveillance of outpatient analgesic safety. This requires obtaining timely, nationally representative surveillance data on outpatient analgesic ADRs.

This study analyzed national data on ADRs of non-opioid and opioid analgesics reported to the Croatian Agency for Medicinal Products and Medical Devices (HALMED) from 2007 to 2014. Study aims were to examine prevalence of reported ADRs of analgesics, frequency of ADRs for various analgesics, where the reports mostly come from, in which patients, types of ADRs and frequency of serious ADRs of analgesics in a national register.

\section{Methods}

\section{Study design}

This was a retrospective study.

\section{Data collection}

Information about ADRs of analgesics were obtained from the Croatian national authority for pharmacovigilance Croatian Agency for Medicinal Products and Medical Devices (HALMED). Data concerning the suspected ADRs were coded into the related Preferred Term and System Organ Class (SOC) using the Medical Dictionary for Drug Regulatory Affairs (MedDRA) adverse drug reaction terminology. ADR reports received in the eight-year period from the beginning of 2007 to the end of 2014 were analyzed.
The following data were collected: type of $\mathrm{ADR}$, year of the ADR report, generic name of a drug, type of report, whether ADR was serious ADR or not), institution in which $A D R$ was reported, the qualifications of the person who reported ADR, patient's date of birth, age group and sex. ADRs were considered serious, as defined by European Medicines Agency (EMA) if they met one of the following criteria: the ADR resulted in death, the ADR was life threatening, the $A D R$ required inpatient hospitalization or prolongation of existing hospitalization, the ADR resulted in persistent or significant disability/incapacity, the ADR was a congenital anomaly/birth defect, or the ADR was another important medical event in accordance to important medical event (IME) list provided by EMA. For each drug we calculated percent of serious ADRs from the total number of ADRs reported for that drug. Collected data were anonymized, and any personal information removed, so patients could not be identified.

\section{Ethics statement}

The study did not include any data collection directly from patients. Instead, anonymized registry data were analyzed.

\section{Statistical analysis}

All data were inserted into the electronic spreadsheets and descriptive statistics was calculated using Microsoft Excel (Microsoft Corp., Redmond, WA, SAD).

\section{Results}

During the studied eight-year period (20072014) HALMED received 796 individual reports of ADRs for analgesic medications. The number of ADR reports was continuously increasing during the analyzed period, and in year 2014 this number was three times higher compared to 2007 (Figure 1). 


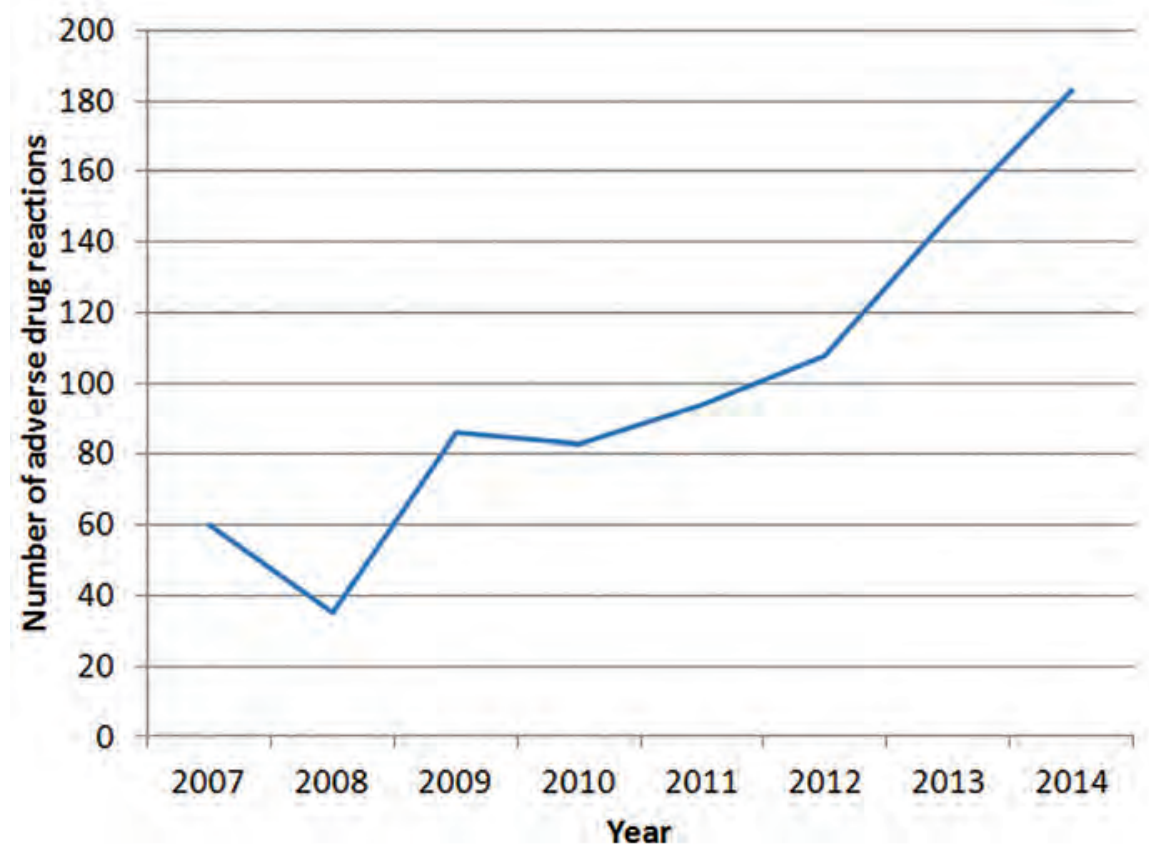

Figure 1 Number of adverse drug reactions of non-opioid and opioid analgesics reported to the Croatian Agency for Medicinal Products and Medical Devices (HALMED) from 2007 to 2014.

Most of the ADR reports were filed by physicians $(\mathrm{n}=350 ; 44 \%)$, followed by pharmacists ( $\mathrm{n}=328 ; 41 \%)$, other health professionals $(n=43 ; 5 \%)$ and patients or other non-health professionals $(\mathrm{n}=32 ; 4 \%)$. For 43 reports it was not recorded in HALMED database who sent the ADR report. ADRs were mostly commonly reported in women $(n=352 ; 59 \%)$, compared to men $(n=286$; $36 \%)$. In 32 ADR reports sex of individuals who suffered from ADRs was not specified. Among all reported ADRs of analgesics in the analyzed period, there were 367 (46\%) serious ADRs and 429 (54\%) were characterized as non-serious. Some of the ADR reports referred to ADRs of individual analgesics, while other ADR reports contained various combination of multiple pharmacological interventions. There were ADR reports for 231 drugs or different combinations of various drugs. There were two drugs/combinations with 4 reports, two drugs/combinations with 3 reports, 20 drugs/combinations with 2 reports and 183 drugs/combinations with only one ADR report.

Among the reported ADRs of analgesics, there were 20 analgesics or combinations with analgesics that were reported $\geq 5$ times, and these were analyzed in more detail. Among those 20 there were 4 drug combinations (Table 1).

ADR reports of those 20 analgesics made 597 (75\%) of all ADR reports for analgesics in the analyzed period. Of the 367 ADRs that were categorized as serious, more than half were associated to only 5 drugs. The highest rate of serious ADRs was noted for drug fentanyl; of the 45 reported ADRs for fentanyl, 38 (84\%) were serious. A combination of drugs acetylsalicylic acid (ASA) and clopidogrel hydrosulfate had 7/10 (78\%) serious ADRs, fentanyl citrate 5/7 (71\%), diclofenac potassium 8/12 (67\%) and fixed combination of caffeine/paracetamol/propyphenazone/codeine phosphate sesquihydrate 8/13 (61\%) serious ADRs. 
Table 1 The frequency of adverse drug reactions reported for the 20 most commonly reported analgesics (2007-2014)

\begin{tabular}{ll}
\hline Generic name & $\mathrm{n}(\%)^{*}$ \\
\hline Ibuprofen & $91(11)$ \\
\hline Diclofenac sodium & $83(10)$ \\
\hline Tramadol/Paracetamol & $59(7.4)$ \\
\hline Acetylsalicylic acid & $57(7.2)$ \\
\hline Tramadol & $55(6.9)$ \\
\hline Ketoprofen & $49(6.2)$ \\
\hline Fentanyl & $44(5.5)$ \\
\hline Paracetamol & $27(3.4)$ \\
\hline Dexketoprofen trometamol & $26(3.3)$ \\
\hline Meloxicam & $19(2.4)$ \\
\hline Caffeine/paracetamol/propyphenazone/ & $13(1.6)$ \\
\hline codeine phosphate sesquihydrate & $12(1.5)$ \\
\hline Diclofenac potassium & $11(1.4)$ \\
\hline Piroxicam & $10(1.3)$ \\
\hline Buprenorphine & $10(1.3)$ \\
\hline Acetylsalicylic acid; Clopidogrel hydrogen & $8(1)$ \\
\hline sulphate & $7(0.9)$ \\
\hline Acetylsalicylic acid /Ascorbic acid & $6(0.7)$ \\
\hline Fentanyl citrate & $5(0.6)$ \\
\hline Etoricoxib & $5(0.6)$ \\
\hline Metamizole natrium & \\
\hline Paracetamol/Pseudoephedrine HCl/Ascorbic \\
acid
\end{tabular}

*Adverse drug reactions of medications whose reports were recorded $\geq 5$ times in analyzed period.

\section{Ibuprofen}

Ibuprofen was drug with the most ADR reports $(n=91 ; 11 \%)$, all of them spontaneous, and 37 (41\%) were classified as serious. Number of reports for ibuprofen ADRs kept increasing from $2007(n=3)$ to $2014(n=27)$. Of the 196 ADRs indicated in those reports for ibuprofen, ADRs were most commonly observed on skin and mucosas $(n=83 ; 42 \%)$, with urticaria, erythema, swollen eyelids and angioedema as the most common of those. Nausea and abdominal pain were the most common gastrointestinal ADRs. Suffocation feeling and dyspnea were the most common respiratory ADRs. Hypertension and hemorrhagic shock were the most common cardiovascular ADRs. Among other ADRs hypersensitivity reactions were the most common. All these ADRs were described only in few patients, as indicated in the Supplementary tables in the online appendix of this manuscript.

ADRs linked with erroneous use of drug were mostly due to off-label use of ibuprofen $(n=4)$. Most of these reports were filed by healthcare workers $(n=64 ; 70 \%)$ and pharmaceutical companies $(n=13 ; 14 \%)$. Most of the reported ADRs were observed in adults $(n=56 ; 62 \%)$, followed by elderly $(n=16$; $18 \%)$ and children $(n=6 ; 6,6 \%)$. Patients were mostly women $(n=58 ; 64 \%)$.

\section{Diclofenac sodium}

Diclofenac sodium was the second most common drug linked to ADR reports $(n=83$; $10 \%)$. Its number of ADRs kept increasing throughout the years, from 3 in 2007 to 15 in 2014. Most of the reports were spontaneous $(\mathrm{n}=79 ; 95 \%)$, while the others were reports from the medical literature. There were 41 (49\%) of ADRs of diclofenac sodium that were classified as serious. Most of the ADRs were reported in adults, and mostly in women $(n=49 ; 59 \%)$. In the 83 ADR reports there were a total of 206 different reported ADRs, which is the highest number of different ADRs reported for any single drug analyzed for this period. The ADRs mostly affected skin and mucosa $(n=63 ; 31 \%)$, with edemas, erythema and rash as the most common ADRs in this group. In the gastrointestinal tract the most common ADRs were different kinds of bleeds, nausea and abdominal pain. Suffocation feeling and dyspnea were the most common respiratory ADRs, while hypertension, hypotension and tachycardia were most commonly observed ADRs in the cardiovascular system. Among other ADRs the most common were vertigo and hypersensitivity reactions. Detailed information 
about ADRs of diclofenac sodium are presented in Supplementary tables in the online appendix of this manuscript.

\section{Tramadol/paracetamol combination}

There were 59 (7.4\%) of ADRs reports submitted for tramadol/paracetamol combination, majority of them in the year 2014. They were mostly spontaneous and $12(20 \%)$ were classified as serious. Majority was reported in women $(\mathrm{n}=43 ; 73 \%)$ and in adults $(\mathrm{n}=30$; $51 \%)$. A total of 175 various ADRs were listed in those 59 reports. Most of them were ADRs in gastrointestinal system $\quad(n=58$; $33 \%$ ), with nausea and vomiting being most common. There were 30 (17\%) of ADRs reported in the nervous system, with vertigo as the most common. A number of ADRs affected general condition of patients, with somnolence the most commonly reported $\mathrm{ADR}$ in this group. Confusion was the most common mental health ADR, hyperhidrosis most commonly reported skin-related ADR, while visual impairment and headache were most commonly reported other ADRs. Detailed information about ADRs of tramadol/ paracetamol combination are presented in Supplementary tables in the online appendix of this manuscript.

\section{Acetylsalicylic acid}

ASA was the subject of 57 (7.2\%) ADR reports, containing a total of 116 various ADRs. Number of reported ADRs for ASA kept increasing over the years, just like for other analyzed medications. Of 57 ADR reports for ASA, there were 28 (49\%) serious ADRs. More ADRs were observed in men $(n=30 ; 53 \%)$. Majority of ADRs were reported in adults $(\mathrm{n}=28 ; 49 \%)$ and elderly $(\mathrm{n}=20 ; 35 \%)$. ADRs related to skin and mucosa were most frequent $(n=38 ; 33 \%)$, with urticaria and rash as the most common ADRs in this group. ADRs in gastrointes- tinal systems followed in frequency $(\mathrm{n}=27$; $23 \%$ ), with the most common ADR being GI bleeding. Most common ADRs in the respiratory system were suffocation feeling and dyspnea, and among other ADRs epistaxis. Detailed information about ADRs of ASA are presented in Supplementary tables in the online appendix of this manuscript.

\section{Tramadol}

For tramadol, 55 (6.9\%) of ADR reports were filed; $12(22 \%)$ were classified as serious. All the reports were spontaneous. Most of the ADR reports referred to women $(n=31 ; 56 \%)$ and adult persons $(n=31 ; 56 \%)$. In the 55 reports there were 169 different ADRs listed, most commonly ADRs were related to gastrointestinal system (30\%), where nausea and vomiting were by far the most common. Erythema and hyperhidrosis were most common dermatological ADRs, while vertigo was the most common neurological ADR. In the cardiovascular system palpitations were most frequent ADR. Among ADRs affecting general condition of a patient, dizziness and asthenia were the most common. Detailed information about ADRs of tramadol are presented in Supplementary tables in the online appendix of this manuscript.

For the other drugs, with lower number of reported ADRs detailed description of those ADRs is provided in Supplementary tables in the online appendix of this manuscript.

\section{Discussion}

This study analyzed reports of adverse events associated with analgesics reported to national Croatian authority from 2007 to 2014 . Among 896 reports there were 20 analgesics with $\geq 5$ reports in the 8 -year period and among them top five were ibuprofen, diclofenac sodium, tramadol/paracetamol combination, acetylsalicylic acid and tramadol. 
ADRs are an important cause of morbidity and mortality; it is estimated that they are on the 4-6th place on the list of leading causes of death in the United States annually (10). Therefore, it is important both to encourage reporting of ADRs and to study them systematically. The main limitation in the analysis of ADRs is insufficient reporting and therefore it is important to keep encouraging all stakeholders to report the observed ADRs, in spite of a series of obstacles related to the process that have been identified in earlier studies (10).

It is especially important to closely monitor the effects of new drugs on the market because in this way, after the introduction of the drug on the market, potentially dangerous ADRs can be caught that were not identified in earlier stages of clinical trials. Spontaneous reports can provide signals that are later confirmed by other databases and complaints of patients. An example of these warning signs in recent times is myocardial infarction caused by a drug rofecoxib and heart failure caused by rosiglitazone (11).

Studies related to the promptness of reporting ADRs were not conducted in Croatia and therefore it is difficult to speculate about the accuracy of data, or to what extent the recorded adverse reactions are actually reported to the HALMED. This study was conducted specifically for the period from the year 2007 to 2014 because the HALMED established a comprehensive electronic database of ADRs in 2007. Electronic database allows for a simpler retrieval of data about ADRs and their analysis.

Historically, hospitals have relied on spontaneous reporting of harms related to drugs. This approach systematically underestimates the frequency of ADRs and recovers only a minority of ADRs. This approach is attractive because it is cheap, compared to other methods of data collection. A better approach is to use patient charts to identify ADRs, but for routine use is too expensive
(12). The third approach in finding ADRs is a computer detection. This method generally uses computer data to identify signals that indicate the possible presence of ADRs. Although this approach still involves the use of the chart to confirm the event, it is much cheaper because only a small part of the scale must be reviewed and review can be focused (12).

The key benefit of electronic medical records is that it can be used to detect the frequency of ADRs and to develop methods for reducing the number of such events. Development and maintenance of the computer system of screening involves several steps. The first and biggest step is to collect information about patient in an electronic form. The second step is to apply inquiries, rules or algorithms to find objects with information in accordance with the examined adverse event. The third step is to determine the predictive value, typically, a manual review (12).

This study provides a detailed description of ADR reports of 20 analgesics or analgesic combinations that had more than $5 \mathrm{ADR}$ reports, covering $75 \%(n=597)$ of all reports of ADRs of analgesics in the analyzed period. Certain ADR reports contained multiple ADRs identified in a patient. Therefore, the number of reports in some cases was not proportional to the number of different ADRs that have been listed in the reports. In this study ibuprofen was drug which had the most ADR reports in the analyzed period. However, diclofenac sodium was a drug for which the biggest number of various ADRs was listed, although it had fewer reports compared to ibuprofen. The ADR reports often record multiple different ADRs in the same patient. For the comparison, analysis of spontaneous reports of side effects of Portuguese national unit for pharmacovigilance shows that the unit received 2,408 reports containing a total of 5749 adverse reactions from 2001 to 2013 (13). 
The highest number of ADRs of analgesics in this study was recorded on the skin and mucous membranes. In 11 of 20 drugs skin disorders were the most frequent. Skin disorders were followed by gastrointestinal disturbances. Problems with blood and blood coagulation were seen as the most frequent in the combination of acetylsalicylic acid and clopidogrel, and psychiatric problems during the use of opioid analgesic fentanyl as fentanyl citrate. The only drug for which death as an ADR was recorded was fentanyl; suicide was the most frequently reported ADR for this drug.

We were unable to find similar studies that covered ADRs of analgesics, and therefore we compared some of our data with similar pharmacovigilance studies on national level, which reported ADRs for all drugs. A recent study of Ozcan et al. about ADRs reported on a national level in Turkey did not present drugs in the same way that we did; they reported drugs for which ADRs were reported according to the body system in line with the ATC classification. Based on their study, drugs acting on a nervous system, which would be concordant with analgesics, were responsible for $14 \%$ of the reported drugs, ranking third among all the categories of drugs that were used (14). Bourgeouis et al. analyzed national estimates and characterizations of outpatient adverse drug events in the United States between 1995 and 2005. They showed that non-opioid analgesics were among the three most common drugs warranting a clinic visit due to adverse drug event, and in the emergency department non-opioid analgesics/antipyretics most frequently resulted in adverse drug event (15).

In this study, $46 \%$ of the ADRs were categorized as serious, and most of those serious ADRs were caused by fentanyl, combination of acetylsalicylic acid and clopidogrel hydrogen sulfate, fentanyl citrate, diclofenac potassium and fixed combination of caf- feine/paracetamol/propyphenazon/codeine phosphate sesquihydrate. Portuguese data for the period 2001-2013 show that among spontaneous ADR reports 55\% were categorized as serious ADRs (16). It is possible that serious ADRs make such a big percent of ADRs because of their gravity - non-serious ADRs could be perceived as less important by the healthcare workers. Different results were reported from an Australian study in 2013, which investigated medicines causing ADRs in patients older than 45 years. Studies were based on survey data collected by family physicians. ADRs were most commonly caused by opioid analgesics. Half of the patients had mild ADRs, $42 \%$ had moderate and $12 \%$ severe ADRs. Five percent of patients were hospitalized due to most recent ADR (17). These differences in the percentages of serious ADRs may be partly due to different categorizations of adverse events. In our study we analyzed data based on two pre-existing categories from the HALMED database, including serious and non-serious ADRs. However, in the Australian study there were four categories of ADRs - mild, moderate, severe and death (17).

A French study published in 2015 found that more than half of cases were serious and led to hospitalization. It was concluded that in $95 \%$ of cases it was possible to prevent those ADRs. The main factor which can be influenced to prevent adverse reactions is careless recommendations for analgesics use and wrong prescribing (17). A 2003 study found that $38 \%$ of ADRs were serious and it was estimated that $28 \%$ of all identified drug ADRs could be prevented. Most of the errors occurred in prescribing and monitoring patient therapy. Adherence errors were also frequent (18). In Croatia there is a network of pain clinics, that employ pain specialists that could be consulted for adopting interventions to prevent such ADRs (19).

In a national study which was conducted in the US in 2003, it was concluded that 
many patients have adverse reactions during transition from hospital to home. According to the study, approximately one out of 5 patients has experienced an adverse event during the transition from hospital to home (20). The fact that people who are leaving hospital are more vulnerable to appearance of ADRs than other patients tells us that some populations are more vulnerable than others when it comes to ADRs. The study also came to the conclusion that one third of adverse events could be prevented. Another third was inevitable, but their weight could be reduced if there were previously implemented corrective actions. These results indicate that there are four areas of potential ADR prevention measures: assessment of patients at the time of discharge, teaching patients about medicines, ADRs and what to do if the specific problems develop, improving therapy monitoring and improving monitoring of the patient's global condition (20). Another report concluded that a larger number of ADRs could be prevented in the elderly because of complexity of their clinical presentation. The side effects and medication errors are the main target in the prevention of adverse events (21). It has been shown that a decision tree model for analysis of ADRs to discover combinations of multiple risk factors that would increase the risk; therefore, such tools could be used in clinical practice (22).

Additionally, patients are prone to selfmedication, and it has been found that sharing of prescription analgesics between patients is common behavior that is mostly viewed positively by patients and even some physicians $(23,24)$. Therefore, educational interventions targeted to patients that will aim to reduce sharing of prescription analgesics to others may help in reducing ADRs.

In this study $96 \%$ of all ADRs reports of analgesics, reports were collected through forms that were filled out by health care workers or patients, i.e. they were spontaneous. Alsham- mari et al. published in 2015 an analysis of the integrity of ADR reports for all medicines in Saudi Arabia and reported for the analyzed period that out of a total of 14,873 ADR reports, $80 \%$ were spontaneous (13).

In this study $42 \%$ of the ADR reports were sent by pharmacists and $43 \%$ by physicians; $39 \%$ of reports were submitted at a pharmacy. These numbers indicate crucial importance of the pharmacist and pharmacies in recording ADRs. A study about social impacts on US pharmacists related to ADRs reporting showed that the majority of respondents intends to report serious adverse reactions, and that the most important influences for submitting ADR report are those coming from the referent regulatory body which registers ADRs, as well as from patients, pharmacy and hospital administrators. Being a woman, having less work experience and better understanding the process of reporting were associated with a greater intention for ADRs reporting (25).

US study published in 2003, with 377 pharmacists included, showed that most of the pharmacists (68\%) had never reported an adverse reaction to the regulatory body. Most pharmacists (66\%) thought that they do not have sufficient knowledge about the process of ADR reporting. On the assessment of knowledge they showed knowledge in the range of $56-96 \%$. The conclusion was that pharmacists should have more education, awareness and practice related to the reporting of ADRs (26).

A study published in Croatia in 2010 analyzed pharmacists' awareness about importance of knowledge about ADRs, pharmacists' knowledge about the pharmacovigilance system in Croatia and pharmacists' personal reasons for not reporting ADRs. The study included 471 pharmacists, mostly employed in the pharmacies. The results showed that pharmacists have sufficient knowledge about ADRs and legal obligation to report $\mathrm{ADR}$, but despite that, number of 
ADR reports made by pharmacists was meager. After attending workshop provided by the HALMED for a target group of pharmacists, an increase in the number of ADRs reported made by pharmacists was observed, from the $0.6 \%$ before 2006 to $22 \%$ in the first 6 months of 2006. This study showed that education of pharmacists can increase the number of reports of ADRs. When they were asked for the reasons for not reporting ADRs, pharmacists indicated that they usually see ADRs which are already known, and that the patient was using more drugs at the same time and because of that it was not possible to establish an association between certain ADRs and a particular drug (27). Some of the pharmacists stated that they do not know how to report an adverse reaction. Pharmacists have a key role in pharmacovigilance (28) and therefore it is important to invest in their knowledge and skills related to the adverse reaction reports, which are legal obligation.

Regarding causality, HALMED uses the World Health Organization - Uppsala Monitoring Centre (WHO-UMC) system for causality assessment. This system has been developed together with national centers that participate in the Programme for International Drug Monitoring and is designed as a practical tool for assessing case reports. That is combined assessment that takes into account the clinical-pharmacological aspects of the case history and the quality of the documentation of the observation. The method then gives guidance to the general arguments which should be used to select one category over another. The WHO-UMC system recognizes six different causality categories: certain, probable/likely, possible, unlikely, conditional/unclassified and unassessable/unclassifiable. For the purpose of this study, we used only cases which were classified in first four categories.

\section{Limitations}

Limitations of our study include lack of causality assessment. For example, ASA is an antiinflammatory drug, but when associated with clopidogrel its therapeutic indication is as an antiplatelet drug. Therefore, ADRs related to blood and blood coagulation problems could be due to an interaction between ASA and clopidogrel, which makes causality assessment difficult. Additional such associations and cofounding variables are possible and reported ADRs may or may not be associated with the analgesics in question.

\section{Conclusion}

In conclusion, number of ADR reports for analgesics is continuously increasing and a considerable number of them refers to serious ADRs. More research in this field is necessary so that health care professionals and patients can choose the appropriate therapy that will cause less ADRs. To keep better track of medications and ADRs it is necessary to educate and encourage health professionals and patients in reporting side effects.

\footnotetext{
What is already known on this topic

Analgesics are widely used and it can be expected that with the increased use one can expect increased number of adverse drug reactions of analgesics. Large increases in the use of both opioid and non-opioid analgesics were observed worldwide.
}

\section{What this study adds}

This study analyzed reports of adverse drug reactions (ADRs) associated with analgesics reported to national Croatian authority from 2007 to 2014. Among 896 reports there were 20 analgesics with $\geq 5$ reports in the 8-year period and among them top five were ibuprofen, diclofenac sodium, tramadol/ paracetamol combination, acetylsalicylic acid and tramadol. We found that the number of ADR reports in Croatia is continuously increasing and a considerable number of them refers to serious ADRs. To keep better track of medications and ADRs it is necessary to educate and encourage health professionals and patients in reporting side effects.

Acknowledgements: This study is based on an 112 page research thesis of the first author (maiden name: Petra Jurkic), which was prepared and defended in 
Croatian language at the University of Split School of Medicine, Split, Croatia.

Authors' contributions: Conception and design: LP; Acquisition, analysis and interpretation of data: PS, DK and LP; Drafting the article: PS, DK and LP; Revising it critically for important intellectual content: PS, DK and LP; Approved final version of the manuscript: PS, DK and LP.

Conflict of interest: The authors declare that they have no conflict of interest.

\section{References}

1. Shojania KG, Marang-van de Mheen PJ. Temporal trends in patient safety in the Netherlands: reductions in preventable adverse events or the end of adverse events as a useful metric? BMJ Qual Saf. 2015;24(9):541-4.

2. Pham JC, Aswani MS, Rosen M, Lee H, Huddle $\mathrm{M}$, Weeks $\mathrm{K}$, et al. Reducing medical errors and adverse events. Annu Rev Med. 2012;63:447-63.

3. Zhou YJ, Boudreau DM, Freedman AN. Trends in the use of aspirin and nonsteroidal anti-inflammatory drugs in the general US population. Pharmacoepidemiol Drug Saf. 2014;23(1):43-50.

4. Krnic D, Anic-Matic A, Dosenovic S, Draganic P, Zezelic S, Puljak L. National consumption of opioid and nonopioid analgesics in Croatia: 20072013. Ther Clin Risk Manag. 2015;11:1305-14.

5. Hamunen K, Laitinen-Parkkonen P, Paakkari P, Breivik H, Gordh T, Jensen NH, et al. What do different databases tell about the use of opioids in seven European countries in 2002? Eur J Pain. 2008;12(6):705-15.

6. De Conno F, Ripamonti C, Brunelli C. Opioid purchases and expenditure in nine western European countries: 'are we killing off morphine?'. Palliat Med. 2005;19(3):179-84. Erratum in: Palliat Med. 2005;19(8):663.

7. Clarke M, Chalmers I. Discussion sections in reports of controlled trials published in general medical journals: islands in search of continents? JAMA. 1998;280(3):280-2.

8. Gudin JA. The changing landscape of opioid prescribing: long-acting and extended-release opioid class-wide Risk Evaluation and Mitigation Strategy. Ther Clin Risk Manag. 2012;8:209-17.

9. Moore N, Charlesworth A, Van Ganse E, LeParc $\mathrm{JM}$, Jones JK, Wall R, et al. Risk factors for adverse events in analgesic drug users: results from the PAIN study. Pharmacoepidemiol Drug Saf. 2003;12(7):601-10.
10. Mirbaha F, Shalviri G, Yazdizadeh B, Gholami K, Majdzadeh R. Perceived barriers to reporting adverse drug events in hospitals: a qualitative study using theoretical domains framework approach. Implement Sci. 2015;10:110.

11. Wahab IA, Pratt NL, Kalisch LM, Roughead EE. Comparing time to adverse drug reaction signals in a spontaneous reporting database and a claims database: a case study of rofecoxib-induced myocardial infarction and rosiglitazone-induced heart failure signals in Australia. Drug Saf. 2014;37(1):53-64.

12. Bates DW, Evans RS, Murff H, Stetson PD, Pizziferri L, Hripcsak G. Detecting adverse events using information technology. J Am Med Inform Assoc. 2003;10(2):115-28.

13. Alshammari TM, Al-Kathiri WH, Le Louet $H$, Aljadhey HS. Completeness of adverse drug reactions reports of the Saudi adverse event reporting system. Saudi Med J. 2015;36(7):821-8.

14. Ozcan G, Aykac E, Kasap Y, Nemutlu NT, Sen E, Aydinkarahaliloglu ND. Adverse Drug Reaction Reporting Pattern in Turkey: Analysis of the National Database in the Context of the First Pharmacovigilance Legislation. Drugs Real World Outcomes. 2016;3(1):33-43.

15. Bourgeois FT, Shannon MW, Valim C, Mandl KD. Adverse drug events in the outpatient setting: an 11-year national analysis. Pharmacoepidemiol Drug Saf. 2010;19(9):901-10.

16. Batel-Marques F, Mendes D, Alves C, Penedones A, Dias P, Martins A, et al. Pharmacovigilance in Portugal: Activity of the Central Pharmacovigilance Unit [in Portuguese]. Acta Med Port. 2015;28(2):222-32.

17. Berreni A, Montastruc F, Bondon-Guitton E, Rousseau V, Abadie D, Durrieu G, et al. Adverse drug reactions to self-medication: a study in a pharmacovigilance database. Fundam Clin Pharmacol. 2015;29(5):517-20.

18. Gurwitz JH, Field TS, Harrold LR, Rothschild J, Debellis K, Seger AC, et al. Incidence and preventability of adverse drug events among older persons in the ambulatory setting. JAMA. 2003;289(9):1107-16.

19. Fidahic M, Dogan K, Sapunar D, Puljak L. National survey of pain clinics in Croatia: Organization and services. Acta Med Acad. 2015;44(1):18-30.

20. Forster AJ, Murff HJ, Peterson JF, Gandhi TK, Bates DW. The incidence and severity of adverse events affecting patients after discharge from the hospital. Ann Intern Med. 2003;138(3):161-7.

21. Thomas EJ, Brennan TA. Incidence and types of preventable adverse events in elderly patients: 
population based review of medical records. BMJ. 2000;320(7237):741-4.

22. Imai S, Yamada T, Kasashi K, Kobayashi M, Iseki K. Usefulness of a decision tree model for the analysis of adverse drug reactions: Evaluation of a risk prediction model of vancomycin-associated nephrotoxicity constructed using a data mining procedure. J Eval Clin Pract. 2017 May 23. Epub ahead of print.

23. Markotic F, Puljak L. Risks associated with borrowing and sharing of prescription analgesics among patients observed by pain management physicians in Croatia: a qualitative study. J Pain Res. 2016;9:1143-51.

24. Markotic F, Vrdoljak D, Puljiz M, Puljak L. Risk perception about medication sharing among patients: a focus group qualitative study on borrowing and lending of prescription analgesics. J Pain Res. 2017;10:365-74.
25. Gavaza P, Brown CM, Lawson KA, Rascati KL, Steinhardt M, Wilson JP. Effect of social influences on pharmacists' intention to report adverse drug events. J Am Pharm Assoc. 2012;52(5):622-9.

26. Gavaza P, Brown CM, Lawson KA, Rascati KL, Wilson JP, Steinhardt M. Texas pharmacists' knowledge of reporting serious adverse drug events to the Food and Drug Administration. J Am Pharm Assoc. 2011;51(3):397-U135.

27. Ivancicevic I. The Study of Adverse Drug Reactions Reporting Features in Pharmacovigilance System in Croatia [Diploma thesis; in Croatian]. Zagreb: Faculty of Pharmacy and Biochemistry, University of Zagreb; 2010 [updated 2011 Dec 16; cited 2017 Oct 9]. Available from: https://bib.irb. $\mathrm{hr} /$ prikazi-rad? \&rad=496595.

28. Sanchez I, Amador C, Plaza JC, Correa G, Amador R. Assessment of an active pharmacovigilance system carried out by a pharmacist [in Spanish]. Rev Med Chile. 2014;142(8):998-1005. 